\title{
Characteristic classes associated to Q-bundles
}

\author{
Alexei Kotov 1, Thomas Strobl2 2 \\ ${ }^{1}$ Laboratoire de Mathematiques, Universite du Luxembourg \\ 2 Institut Camille Jordan, Universite Claude Bernard Lyon 1
}

\begin{abstract}
A Q-manifold is a graded manifold endowed with a vector field of degree one squaring to zero. We consider the notion of a Q-bundle, that is, a fiber bundle in the category of Q-manifolds. To each homotopy class of "gauge fields" (sections in the category of graded manifolds) and each cohomology class of a certain subcomplex of forms on the fiber we associate a cohomology class on the base. Any principal bundle yielding canonically a Q-bundle, this construction generalizes Chern-Weil classes. Novel examples include cohomology classes that are locally the de Rham differential of the integrands of topological sigma models obtained by the AKSZ-formalism in arbitrary dimensions. For Hamiltonian Poisson fibrations one obtains a characteristic 3-class in this manner. We also relate to equivariant cohomology and Lecomte's characteristic classes of exact sequences of Lie algebras.
\end{abstract}

MSC classification: 58A50, 55R10, 57R20, 81T13, 81T45,

Keywords: Q-manifolds, gauge theories, characteristic classes

\section{Introduction}

1.1 The notion of a Q-manifold provides a general framework for studying gauge theories within the Batalin-Vilkovisky formalism [20]. It is particularly useful in the context of topological sigma models [1]. A Q-manifold, also known as a differential-graded (dg) manifold, is a graded manifold $\mathcal{M}$ endowed with a degree one vector field $Q$ which satisfies the equation $[Q, Q] \equiv 2 Q^{2}=0$. Hereafter we suppose that the algebra of functions on $\mathcal{M}$ is non-negatively graded, unless the contrary is stated. We say that $\mathcal{M}$ is a $\mathrm{Qp}$ manifold, if the algebra of functions is locally generated in degree up to $\mathrm{p}$.

1.2 Let us enumerate some basic examples of Q-manifolds appearing in the literature.

(1) A Lie algebra $\mathfrak{g}$ considered as a purely odd manifold of degree one, denoted as $\mathfrak{g}[1]$. The algebra of functions is naturally isomorphic to $\Lambda^{\bullet} \mathfrak{g}^{*}$, the cochain complex of $\mathfrak{g}$, and the Q-field is nothing but the Chevalley-Eilenberg differential.

(2) A Lie algebroid $E$ with the degree of fibers shifted by one, denoted as $E$ [1]. The algebra of functions is identified with $\Gamma\left(\Lambda^{\bullet} E^{*}\right)$ and the Q-field is the canonical differential. Moreover, every Q1-manifold is necessarily of the form $E[1]$ for a certain Lie algebroid [22]. In general, a homological vector field of degree one on an arbitrary graded vector bundle determines an $L_{\infty}$-algebroid structure by use of multi-derived brackets, cf., e.g., [23].

\footnotetext{
${ }^{1}$ e-mail address: Alexei.Kotov @ uni.lu

${ }^{2}$ e-mail address: Strobl @ math.univ-lyon1.fr
} 
(3) A PQ-manifold: This is a graded manifold $\mathcal{S}$ supplied with a symplectic form $\omega$ of degree $\mathrm{p}$ and a function $Q$ of degree $\mathrm{p}+1$, which obeys the equation of self-commutativity with respect to the non-degenerate Poisson bracket determined by $\omega$. The Q-field is the hamiltonian vector field of $Q \cdot \sqrt[3]{3}$

1.3 A morphism of Q-manifolds (Q-morphism) is a degree preserving map $\phi$, the pullback of which commutes with the corresponding homological vector fields, considered as super derivations of functions, i.e. the following chain property holds: $Q_{1} \phi^{*}=\phi^{*} Q_{2}$. A morphism of Q1-manifolds is nothing but the morphism of the corresponding Lie algebroids ([22]; cf. [2] for a proof of equivalence with the original definition of Lie algebroid morphisms given in [14]). Given a smooth map of two manifolds $m: M \rightarrow N$, its pushforward defines a Q-morphism $m_{*}: T[1] M \rightarrow T[1] N$ of the tangent bundles, where a Qstructure on the odd tangent bundle of a manifold is determined by the de Rham operator regarded as a homological vector field by use of the identification $C^{\infty}(T[1] M) \simeq \Omega^{\circ}(M)$.

Apparently, a composition of two Q-morphisms is again a Q-morphism, so there is a well-defined category of Q-manifolds. A Q-bundle, a fiber bundle in this category, is a surjective morphism of the total space to the base of the bundle, satisfying an additional requirement of local triviality: a bundle is built from direct products of local charts on the base and a fixed fiber glued by a transition cocycle of "gauge transformations"- as will be detailed further in section 2 below. By a "gauge field" $\varphi$ in a Q-bundle $\pi: \mathcal{M} \rightarrow \mathcal{M}_{1}$ we mean a section of the underlying bundle of graded manifolds. In general we do not assume that a section is a Q-morphism! (The existence of such a section imposes a certain constraint on the bundle).

Some examples of these constructions are the following ones, with the third one providing the relation to ordinary gauge theories:

(1) The product of two Q-manifolds is again a Q-manifold and the projection to each factor produces a (trivial) Q-bundle structure.

(2) A fiber bundle $p: M \rightarrow M_{1}$ determines a natural "non-linear" example of a Q-bundle by use of the the push-forward map: $p_{*}: T[1] M \rightarrow T[1] M_{1}$. Apparently, the pushforward of any section of $p$ is a section of $p_{*}$, which is, indeed, a morphism of the corresponding Q-manifolds.

(3) Given a principal G-bundle $p: P \rightarrow M$, we construct a Q-bundle in the following way: As total space we take the quotient of $T[1] P / G$, where the group action of $G$ on $P$ is lifted in the canonical way and the quotient by $G$ can be considered as a bundle over $M$. Using the push-forward $p_{*}$ of $p$, on the other hand, we obtain a (degree-preserving) map to $T[1] M$, the base of the Q-bundle. Both spaces are canonically equipped with the de Rham differential (in the first case restricted to $G$-invariant differential forms on $P$ ). This construction is known as the Atiyah algebroid of $P$, which is a particular Lie algebroid ( $T[1] P$ is obviously a degree one $Q$-manifold). A connection in $P$ provides a lift of tangent vectors on $M$ to tangent vectors of $P$; by its equivariance w.r.t. the $G$-action this corresponds precisely to a bundle map $\varphi: T M \rightarrow T P / G$, i.e. a section of the bundle

\footnotetext{
${ }^{3}$ For $p>1$ a PQ-manifold is equivalent to a Q-manifold with compatible degree $\mathrm{p}$ symplectic form [18].
} 
$p_{*}: T[1] P / G \rightarrow T[1] M$. As we will see in detail in section 2 below, the connection is flat, iff $\varphi$ is a Q-morphism.

(4) A transitive Lie algebroid $E \rightarrow M$, in generalization of an Atiyah algebroid: By definition this is a Lie algebroid with surjective anchor $\rho$, yielding the short exact sequence

$$
0 \rightarrow \underline{\mathfrak{g}} \rightarrow E \stackrel{\rho}{\rightarrow} T M \rightarrow 0,
$$

where $\underline{g}$ is a bundle of Lie algebras defined by the kernel of $\rho$. We restrict to the case that any of the fibers $\operatorname{ker} \rho$ is isomorphic to a single Lie algebra $\mathfrak{g} 4$. This then yields a Q-bundle $\rho: E[1] \rightarrow T[1] M$ with typical fiber $\mathrm{g}[1]$. Note that since $\rho$ is a morphism of Lie algebroids, the projection is a Q-morphism. In this particular case, a gauge field $\varphi: T[1] M \rightarrow E[1]$ is a splitting of the exact sequence of Lie algebroids (1.1). It is sometimes also called a "connection" of the transitive Lie algebroid $E$, in generalization of the previous example, and called "flat" in a situation when $\varphi$ is a Q-morphism.

(5) An exact sequence of Lie algebras - cf. example (1) of paragraph 1.2 - is a Q-(fiber)bundle (as defined above) only in the case when it is isomorphic to a direct sum of Lie algebras. We will address this situation at the end of the paper.

(6) More generally than examples (2) - (5), one can consider an exact sequence of Lie algebroids, covering an ordinary fiber bundle; if the total Lie algebroid splits locally into a direct product of fiber and base Lie algebroids, it fits into the definition of Q1-bundles above. We will study particular examples of this, where the fibers are some given PQmanifold and the base a tangent Lie algebroid, considering applications in section 4 below.

1.4 For an arbitrary degree preserving map of Q-manifolds $\varphi: \mathcal{M}_{1} \rightarrow \mathcal{M}_{2}$ the difference $F:=Q_{1} \varphi^{*}-\varphi^{*} Q_{2}$, which we call the "field strength" if $\varphi$ is a (coarse-grained) section of a Q-bundle $\mathcal{M}_{2} \rightarrow \mathcal{M}_{1}$, is non-vanishing in general. It is a degree one derivation of functions on the target taking values in functions on the source

$$
F: C^{\infty}\left(\mathcal{M}_{2}\right) \rightarrow C^{\infty}\left(\mathcal{M}_{1}\right)
$$

for which the following Leibnitz-type property holds:

$$
F(g h)=F(g) \varphi^{*}(h)+(-1)^{\operatorname{deg}(g)} \varphi^{*}(g) F(h), \quad \forall g, h \in C^{\infty}\left(\mathcal{M}_{2}\right) .
$$

Therefore $F$ can be identified with a degree one section of the pull-back bundle $\varphi^{*}\left(T \mathcal{M}_{2}\right)$ over $\mathcal{M}_{1}$ or, as is equivalent, with a degree preserving map $f: \mathcal{M}_{1} \rightarrow T[1] \mathcal{M}_{2}$ covering $\varphi$, as will be further detailed in section 3 below. The graded manifold $T[1] \mathcal{M}$, where $\mathcal{M}$ is a Q-manifold, is a double Q-manifold (or a double Q-algebroid, cf. [13, 24]), i.e. it admits a pair of anti-commuting homological vector fields. It will turn out, cf. Proposition 3.3 below, that $f$ is a Q-morphism if $T[1] \mathcal{M}_{2}$ is endowed with the sum of two canonical Qstructures as differential. Note that in contrast to the Leibnitz property (1.3) of $F$, the map $f^{*}: C^{\infty}\left(T[1] \mathcal{M}_{2}\right) \rightarrow C^{\infty}\left(\mathcal{M}_{1}\right)$ defines a morphism of algebras, and thus, being a chain map, also a map in cohomologies.

\footnotetext{
${ }^{4}$ Note that even under this assumption not every transitive Lie algebroid comes from a principal bundle. Only if this Lie algeroid can be integrated to a Lie groupoid, this is the case.
} 
Using the Bernstein-Leites sign convention, functions on $T[1] \mathcal{M}$ can be identified with differential forms on $\mathcal{M}$. Given a trivialization of a Q-bundle over some open cover of the base, we can always identify a section with a family of degree preserving maps from the local charts to the fiber, which are related by the transition transformations on double overlaps. By means of the pullback of the above map $f$, we obtain a family of chain maps acting from the total complex of differential forms on the fiber to the complex of functions on the open charts, which are different in general on double overlaps. However, applying the collection of chain maps to a differential form on the fiber, which is invariant with respect to the gluing transition functions (or, equivalently, the "gauge transformations") we will call such forms basic-, we obtain a well-defined cocycle on the whole base. This thus provides a map from the cohomology of basic forms on the fiber to the cohomology of forms on the base, cf. Theorem 3.10 below. The construction will be seen to generalize the Chern-Weil formalism of characteristic classes associated to principal bundles.

1.5 Some brief remark on our notation and nomenclature: As we recalled above, a connection in a principal bundle $P$ is in bijection to a splitting in (1.1) of the corresponding Atiyah algebroid $E \rightarrow M$, or, what is the same, a (globally well-defined) section $\varphi$ (in the coarse-grained sense) of a likewise Q-bundle $\rho: E[1] \rightarrow T[1] M$. The map $f$ above can be seen to generalize the curvature of that connection on $P$, moreover. Since, on the other hand, on a Q-bundle $\pi: \mathcal{M} \rightarrow \mathcal{M}_{1}$ one can also discuss (super-) connections and curvatures, we refrained from calling such maps $\varphi$ and $f$ as (generalized) connections and curvatures, respectively. Instead we thus prefer a physics oriented nomenclature in this context, calling $\varphi \mathrm{s}$ "gauge fields" and $f$ s "field strengths"; in the context of $P$, they locally are represented by (Lie algebra valued) 1 -forms and 2-forms $A$ and $F \sim F_{A}$, respectively. Note that in a more general situation $\varphi$ may correspond to a collection of differential forms of different degrees, or, when $\mathcal{M}_{1}$ is not the shifted tangent bundle of some manifold, even not to differential forms at all.

"Gauge transformations" or "gauge symmetries" will turn out to be related also to vertical automorphisms of the Q-bundle (vertical automorphisms of a principal bundle give rise to anchor preserving automorphisms of the Atiyah algebroid), but in general it will be useful to consider only a subset of the latter ones for gluing transformations.

1.6 The paper is organized as follows. In section 2 we discuss the appropriate notion of gauge transformations, generalizing [2], for a Q-bundle and describe their action on the space of gauge fields.

In section 3 we prove the chain property of the map $f$, defined above, and show that the Weil algebra model of characteristic classes is a particular case of our construction. Here we slightly adapt the notion of a basic form, looking at the action of gauge symmetries on $f$, and complete the construction of characteristic classes associated to a section of a Q-bundle. We also prove the obtained cohomological classes are homotopical invariants of such sections.

In section 4 we explain a possible construction of characteristic classes whose cocycles turn out to be locally represented by integrands of the (classical part of) topological AKSZ-type sigma models. In the case of Hamiltonian Poisson fibrations, the typical 
fiber being a Poisson manifold, one obtains a 3-class in de Rham cohomology on the base manifold, which, locally, agrees with the original construction of (the integrand of) the Poisson sigma model in [19]. In section 4 we also address the gauging of Wess-Zumino terms, its relation to equivariant cohomology, and the characteristic classes of [11] within the present framework.

\section{Q-bundles and gauge symmetries}

2.1 In order to explain the notion of gauge fields and gauge symmetries, we start with a simple example of $\mathfrak{g}$-valued 1 -forms on a smooth manifold $M$, where $\mathfrak{g}$ is a Lie algebra. Given $A \in \Omega^{1}(M, \mathfrak{g})$, interpreted as a connection in a trivial bundle $M \times G, \operatorname{Lie}(G)=\mathfrak{g}$, we look at its curvature:

$$
F_{A}:=\mathrm{d} A+\frac{1}{2}[A, A]
$$

The group of $G$-valued functions on $M$ is acting on connections by: $A^{g}=g^{-1} \mathrm{~d} g+$ $\operatorname{Ad}_{g^{-1}}(A)$, where $g^{-1} \mathrm{~d} g$ is the pull-back by $g$ of the (left) Maurer-Cartan form on the Lie group and Ad is the adjoint action. The above transformations correspond to vertical automorphisms of the trivial bundle and are called gauge transformations in the physics literature. Their infinitesimal version is goverend by a $\mathfrak{g}$-valued function $\epsilon$ :

$$
\delta_{\epsilon} A:=\left.\frac{\mathrm{d}}{\mathrm{d} t} A^{\exp (t \epsilon)}\right|_{t=0}=\mathrm{d} \epsilon+[A, \epsilon] .
$$

The condition of flatness, $F_{A}=0$, can be also regarded as Maurer-Cartan equation for $A$.

2.2 Let us adapt this example to the language of dg or Q-manifolds. As we already know, a Lie algebra can be treated as a Q-manifold $\mathfrak{g}[1]$, such that the algebra of functions becomes isomorphic to $\Lambda\left(\mathfrak{g}^{*}\right)$ with the Q-field given by the Chevalley-Eilenberg differential:

$$
\mathrm{d}_{\mathrm{g}}(\alpha)\left(\eta, \eta^{\prime}\right)=-\alpha\left(\left[\eta, \eta^{\prime}\right]\right)
$$

where $\alpha \in \mathfrak{g}^{*}$ and $\eta, \eta^{\prime} \in \mathfrak{g}$. The product of $T[1] M$ and $\mathfrak{g}[1]$ is again a Q-manifold, the Q-structure of which is given by the sum of de Rham and Chevalley-Eilenberg derivations extended to the product in the standard way. A $\mathfrak{g}$-valued 1-form on $M$ can be thought of as a degree preserving map $\varphi: T[1] M \rightarrow \mathfrak{g}[1]$ and its graph as a section of the bundle

$$
T[1] M \times \mathfrak{g}[1] \rightarrow T[1] M .
$$

The pull-back of $\varphi$ is acting as follows: for each $\omega \in \Omega(M), \alpha \in \Lambda^{p}\left(\mathfrak{g}^{*}\right)$ one has

$$
\varphi^{*}(\alpha \otimes \omega)=\alpha(\underbrace{A \wedge \ldots, A}_{p \text { times }}) \wedge \omega
$$

Any $\mathfrak{g}$-valued function $\epsilon$, acting by the contraction $\iota_{\epsilon}$ on $\Omega(M) \otimes \Lambda\left(\mathfrak{g}^{*}\right)$, can be considered as a super-derivation of degree -1 , which super-commutes with $\Omega(M)$. The last property implies that it can be identified with a vertical vector field on the total space of (2.4). 
Proposition 2.3 The following identity holds for each $\omega \in \Omega(M)$ and $\alpha \in \Lambda^{p}\left(\mathrm{~g}^{*}\right)$ :

$$
\begin{aligned}
\left(\mathrm{d} \varphi^{*}-\varphi^{*}\left(\mathrm{~d}+\mathrm{d}_{\mathrm{g}}\right)\right)(\alpha \otimes \omega) & =\sum_{k}(-1)^{k+1} \alpha(A \wedge, \ldots \wedge \overbrace{F_{A}}^{k}, \ldots \wedge A) \wedge \omega \\
\varphi^{*} L_{\epsilon}(\alpha \otimes \omega) & =\sum_{k} \alpha(A, \wedge \ldots, \overbrace{\delta_{\epsilon} A}^{k}, \ldots \wedge, A) \wedge \omega
\end{aligned}
$$

where $L_{\epsilon}$ is the Lie derivative along $\epsilon$, defined as the super-commutator $\left[Q, \iota_{\epsilon}\right]$ and $\delta_{\epsilon} A$ is given by formula (2.2) above.

Proof. Straightforward calculations.

Note that, instead of thinking of an infinitesimal gauge transformation as a flow on the space of connections, we define a vector field on the total space of (2.4), the action of which on the space of connections, regarded as sections of (2.4), can be naturally induced. One may wonder why, though $A$ is extended as a morphism of graded manifolds, its infinitesimal variation is extended (by the Leibnitz rule) as a derivation. Indeed, it is a general fact, adapted to the graded case, that the space of infinitesimal variations (the tangent space) of a smooth map $\psi: M \rightarrow N$ can be identified with the space of sections of the pullback bundle $\psi^{*}(T N)$ or, equivalently, with the space of derivations $\delta: C^{\infty}(N) \rightarrow$ $C^{\infty}(M)$ covering $\psi$ :

$$
\delta\left(h h^{\prime}\right)=\delta(h) \psi^{*}\left(h^{\prime}\right)+(-1)^{\operatorname{deg}(\delta) \operatorname{deg}(h)} \psi^{*}(h) \delta\left(h^{\prime}\right)
$$

for any $h, h^{\prime} \in C^{\infty}(N)$. As it is clear from (2.5), the curvature $F_{A}$ is the only obstruction for $A$ to be a Q-morphism; $A$ gives a flat connection, $F_{A}=0$, iff the corresponding section $\varphi: T[1] M \rightarrow T[1] M \times \mathfrak{g}[1]$ is a Q-morphism.

2.4 It is evident how to generalize the picture described above for connections in a nontrivial vector bundle or its associated principal bundle $P$ : For this purpose we return to example (3) in paragraph 1.3 above, replacing the trivial Q-bundle (2.4) by its Atiyah algebroid $E$, i.e. by

$$
\rho: E[1] \rightarrow T[1] M
$$

where $E=T P / G$ and $\rho=p_{*}$ denotes the anchor map. Then a connection becomes a section of (2.7). An infinitesimal gauge transformation a Lie derivative with respect to some section of $E$ lying in the kernel of $\rho$. The space of such sections is in oneto-one correspondence with the space of vertical vector fields of degree minus one on the Q-bundle $E[1] \rightarrow T[1] M$; the correspondence is given by the contraction, $\iota_{\epsilon}$ for any $\epsilon \in \Gamma(E)$ can be regarded as a vector field on $E[1]$. The gauge transformations are thus generated by $L_{\epsilon} \equiv\left[Q, \iota_{\epsilon}\right]$, where $\epsilon \in \Gamma(\operatorname{ker} \rho)$ or, equivalently, $\rho_{*} \iota_{\epsilon}=0$. Elements $\alpha \otimes \omega \in \Lambda^{p}\left(\mathfrak{g}^{*}\right) \otimes \Omega(M)$, used in Proposition 2.3 above now generalize merely to functions on $E[1]$.

2.5 Now we can describe a general Q-bundle, its gauge transformations and fields. The Lie super-algebra of vector fields on $\mathcal{M}$, denoted as $\mathcal{D}(\mathcal{M})=\oplus_{k} \mathcal{D}^{k}(\mathcal{M})$, is a differential 
graded Lie algebra, the differential of which is given by the adjoint action of $Q: a d_{Q}(X):=$ $[Q, X]$ for $X \in \mathcal{D}(\mathcal{M})$. Indeed, since $Q$ satisfies the "master equation" $[Q, Q]=0$, its double commutator with any vector field vanishes: by the super Jacobi identity one has

$$
a d_{Q}^{2}(X)=[Q,[Q, X]]=\frac{1}{2}[[Q, Q], X] \equiv 0 .
$$

We call a vector field $X \in \mathcal{D}^{0}(\mathcal{M})$ commuting with $Q$ an infinitesimal symmetry, or simply symmetry, of a Q-manifold and a degree zero vector field, which is a commutator of $Q$ with some other vector field, an inner derivation or inner (infinitesimal) symmetry. In the case of a Lie algebra $g[1]$ this agrees with the usual nomenclature, whereas in the case of $T[1] M$ e.g. all symmetries are inner in this sense and correspond to vector fields, i.e. (infinitesimal) diffeomorphisms. By definition, infinitesimal symmetries and inner derivations are cocycles and coboundaries in $\left(\mathcal{D}(\mathcal{M}), a d_{Q}\right)$, respectively. The inclusion "coboundaries $\subset$ cocycles" implies that all inner derivations are infinitesimal symmetries of a Q-manifold. The following identity follows from the super Jacobi identity and the nilpotency of $a d_{Q}$ :

$$
\left[\operatorname{ad}_{Q}(X), a d_{Q}\left(X^{\prime}\right)\right]=a d_{Q}\left(\left[X, X^{\prime}\right]_{Q}\right),
$$

where $\left[X, X^{\prime}\right]_{Q}:=(-1)^{\operatorname{deg} X+1}\left[\operatorname{ad}_{Q}(X), X^{\prime}\right] \equiv\left[[X, Q], X^{\prime}\right]$ is known in mathematics as the derived bracket [9]. The space of vector fields supplied with the derived bracket is an example of a Loday algebra. Note that the derived bracket between two vector fields is not (super) skew-symmetric, unless the vector fields are super-commuting. Obviously, vector fields of degree minus one are closed with respect to the derived bracket. Moreover, as is clear from (2.8), but also can be verified directly, its image by $a d_{Q}$ is a (super) Lie subalgebra in $\mathcal{D}^{0}(\mathcal{M})$.

Definition 2.6 A subgroup $H$ of degree preserving maps is called a subgroup of automorphisms (respectively, inner automorphisms), if its Lie algebra consists of infinitesimal symmetries (respectively, inner infinitesimal symmetries).

Before writing the general definition, let us examine once more the trivial example, which is a brick underlying a global design. Suppose $\mathcal{M}=\mathcal{N} \times \mathcal{F}$ is a product of two Qmanifolds $\mathcal{N}$ and $\mathcal{F}$ and $\pi: \mathcal{M} \rightarrow \mathcal{N}$ is a bundle given by the projection to the first factor. It is obvious that the space of vertical vector fields can be identified with sections of the pull-back of $T \mathcal{F}$ w.r.t. the second projection.

Proposition 2.7 Let $\mathcal{G}$ be a graded Lie subalgebra of vector fields on $\mathcal{F}$, closed under the derived bracket. Then the space of functions on $\mathcal{N}$ taking values in $\mathcal{G}$ is a Lie subalgebra of vertical vector fields closed under the derived bracket on the total space $\mathcal{N} \times \mathcal{F}$.

Proof. Let us take an arbitrary $Y \in C^{\infty}(\mathcal{N}, \mathcal{G})$, which can always be written as a linear combination $Y=\sum_{j} b^{j} Y_{j}$, where $b^{j}$ are functions on the base and $Y_{j} \in \mathcal{G}$. Then the inner derivative generated by $Y$ on the total space is

$$
a d_{Q}(Y)=\left[Q_{1}+Q_{2}, Y\right]=\sum_{j} Q_{1}\left(b^{j}\right) Y_{j}+(-1)^{\operatorname{deg}\left(b^{j}\right)} b^{j} a d_{Q_{2}}\left(Y_{j}\right) .
$$


For any $X=\sum_{i} a^{i} X_{i}$, its derived bracket with $Y$ is again a function taking values in $\mathcal{G}$ :

$$
\begin{array}{r}
{[X, Y]_{Q}=\sum_{i, j}(-1)^{\operatorname{deg}\left(X_{i}\right)+\operatorname{deg}\left(a^{i}\right)+1}\left(\left[Q_{1}\left(a^{i}\right) X_{i}+(-1)^{\operatorname{deg}\left(a^{i}\right)} a^{i} a d_{Q_{2}}\left(X_{i}\right), b^{j} Y_{j}\right]\right)=} \\
\sum_{i, j}\left((-1)^{\operatorname{deg}\left(a^{i}\right)+\operatorname{deg}\left(X_{i}\right)\left(\operatorname{deg}\left(b^{j}\right)+1\right)+1} Q_{1}\left(a^{i}\right) b^{j}\left[X_{i}, Y_{j}\right]+(-1)^{\left(\operatorname{deg}\left(X_{i}\right)+1\right) \operatorname{deg}\left(b^{j}\right)} a^{i} b^{j}\left[X_{i}, Y_{j}\right]_{Q}\right),
\end{array}
$$

which implies that $\mathcal{G}$ is a Loday algebra with respect to the total Q-structure.

Let us use the notation for the following Lie algebra of vector fields on the total space:

$$
\mathcal{G}^{\prime}:=\operatorname{ad}_{Q}\left(C^{\infty}(\mathcal{N}, \mathcal{G})\right) \cap \mathcal{D}^{0}(\mathcal{N} \times \mathcal{F}) .
$$

Is is not a surprise for us that $\mathcal{G}^{\prime}$ consists of vertical vector fields. Indeed, suppose we are given $X \in \mathcal{G}^{\prime}$, then there exists some element $\epsilon \in \mathcal{G}$ such that $X=[Q, \epsilon]$. Both of two vector fields in the commutator are $\pi$-projectable, since $\pi_{*}(Q)=Q_{1}$ and $\pi_{*}(\epsilon)=$ 0 , thus $\pi_{*}(X)=0$. It well-known that exponentiating a vertical vector field (at least locally), we obtain a fiber-wisely acting automorphism, i.e. an automorphism $\Psi$ satisfying $\pi \circ \Psi=\pi$. Apparently, the set of fiber-wisely acting automorphisms is a subgroup of all automorphisms of a bundle and a composition of $\Psi$ with any section of $\pi$ is again a section. In this way we can now return to the general, nontrivial bundle situation, formulating the following

Definition 2.8 A $Q$-bundle $\pi: \mathcal{M} \rightarrow \mathcal{M}_{1}$ with typical fiber $\mathcal{F}$ and a holonomy algebra $\mathcal{G} \subset \mathcal{D}^{<0}(\mathcal{F})$ (a chosen graded Lie subalgebra of vector fields on $\mathcal{F}$, closed under the derived bracket) is a surjective $Q$-morphism, satisfying the local triviality condition: there exists an open cover $\left\{\mathcal{U}_{i}\right\}$ of $\mathcal{M}_{1}$ such that the restriction of $\pi$ to each $\mathcal{U}_{i}$ admits a trivialization $\pi^{-1}\left(\mathcal{U}_{i}\right) \simeq \mathcal{U}_{i} \times \mathcal{F}$ in the category of $Q$-manifolds and this trivialization is glued over $\mathcal{U}_{i} \cap \mathcal{U}_{j}$ by inner automorphisms which belong to $\exp \left(\mathcal{G}^{\prime}\right)$ where $\mathcal{G}^{\prime}$ is as in (2.10) with $\mathcal{N}=\mathcal{U}_{i} \cap \mathcal{U}_{j}$.

A gauge field is a section of $\pi$ in the category of graded manifolds, that is, a degree preserving map $\varphi: \mathcal{M}_{1} \rightarrow \mathcal{M}$ which obeys $\pi \circ \varphi=$ Id. A gauge transformation (an infinitesimal gauge transformation) is a fiber-wisely acting inner automorphism (vertical inner derivation) of the total space of $\pi$.

Concatenating a section with a vertical automorphism of the total space, one obtains an action of the group of gauge transformations on the space of sections. In generalization of (2.6) one then has

Proposition 2.9 Given a gauge field $\varphi$ and an infinitesimal gauge transformation $X=$ $a d_{Q}(Y)$, the variation of $\varphi$ along $X$ can be identified with the derivation $\left(\delta_{X} \varphi\right)^{*}:=\varphi^{*} X$ covering $\varphi$.

\section{Field strength and characteristic classes}

3.1 The obvious role of curvature arises from the fact that it can be regarded as an obstruction for a map to satisfy the Maurer-Cartan equation: this motivating example was 
considered in the previous section. In the case of general Q-manifolds, the set of maps between them does not admit a vector space structure any more, so rather than using the language of Lie brackets, we dealt with differential graded algebras as a more general tool. We have also stressed earlier that the Maurer-Cartan (or zero curvature) equation is a particular example of the chain property $Q_{1} \varphi^{*}-\varphi^{*} Q_{2}=0$. The operator [2]

$$
F:=Q_{1} \varphi^{*}-\varphi^{*} Q_{2},
$$

called the field strength, being a replacement of the curvature, is a degree one derivation of functions on the target manifold $\mathcal{M}_{2}$ taking values in functions in the source manifold $\mathcal{M}_{1}$ and covering $\varphi^{*}$. (Here $\varphi: \mathcal{M}_{1} \rightarrow \mathcal{M}_{2}$ is a morphism of graded manifolds, corresponding to a gauge field in a trivial bundle. In the case of a non-trivial bundle, $\mathcal{M}_{2}=\mathcal{M}$, the total space of a Q-bundle $\pi: \mathcal{M} \rightarrow \mathcal{M}_{1}$, and the gauge field $\varphi$ satisfies $\pi \circ \varphi=$ Id.) We have evidence, for instance from the Yang-Mills theory, that the curvature is a meaningful object itself, so one can expect a similar importance of the "field strength" 5 It is advantageous to reformulate the operator (3.1) somewhat, so that the Leibnitz-property (1.3) is replaced by a morphism of algebras (appropriate polynomials should go into polynomials of field strengths). To this end we regard the following non-commuting diagram,

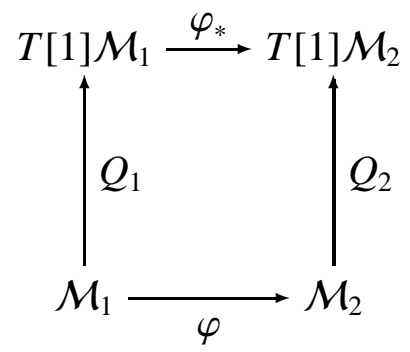

where the homological vector fields are considered as maps and, being of degree one, the tangent bundle was shifted in degree so that the maps are morphisms of graded manifolds. Now one notes that both ways from $\mathcal{M}_{1}$ to $T[1] \mathcal{M}_{2}$ end in the same fiber over $\mathcal{M}_{2}$; thus it is meaningful to define the difference $f: \varphi_{*} \circ Q_{1}-Q_{2} \circ \varphi$, covering $\varphi$

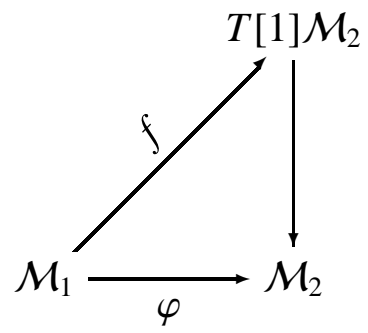

It is easy to convince oneself that for any function $h \in C^{\infty}\left(\mathcal{M}_{2}\right)$ and any $\alpha, \beta \in C^{\infty}\left(T[1] \mathcal{M}_{2}\right)$ one has

$$
f^{*}(h)=\varphi^{*}(h), \quad f^{*}(\mathrm{~d} h)=F(h), \quad f^{*}(\alpha \beta)=f^{*}(\alpha) f^{*}(\beta) .
$$

We shall see below that $f$ is a Q-morphism, if $T[1] \mathcal{M}_{2}$ is endowed with a suitable Qstructure.

\footnotetext{
${ }^{5}$ This idea was implemented e.g. in [21].
} 
3.2 For any graded manifold $\mathcal{M}$, the algebra of functions on $T$ [1] $\mathcal{M}$ admits a simple description as the algebra of super differential forms $\Omega(\mathcal{M})$ (according to the BernsteinLeites sign convention [12]). More precisely, the algebra of forms is generated by $h$ and $\mathrm{d} h$ for all functions $h$ with the following relations:

$$
h \mathrm{~d} h^{\prime}=(-1)^{\operatorname{deg}(h)\left(\operatorname{deg}\left(h^{\prime}\right)+1\right)} \mathrm{d} h^{\prime} h, \quad \mathrm{~d}\left(h h^{\prime}\right)=\mathrm{d} h h^{\prime}+(-1)^{\operatorname{deg}(h)} h \mathrm{~d} h^{\prime} .
$$

This algebra is naturally bi-graded by degrees of functions and orders of forms, such that $\mathrm{d}$, the super-version of the de Rham differential, becomes a (nilpotent) operator of degree zero with respect to the first grading and of degree one with respect to the second grading. The super-commutativity relations are subordinated to the total grading which is the sum of the two. A vector field $X$ of degree $p$ gives a contraction of degree $p-1$ acting as follows:

$$
\iota_{X}(f \mathrm{~d} h)=(-1)^{\operatorname{deg}(f)(\operatorname{deg}(X)+1)} f X(h) .
$$

The super Lie derivative along $X$, an operator of degree $\mathrm{p}$, is defined as the commutator

$$
\mathcal{L}_{X}:=\iota_{X} \mathrm{~d}+(-1)^{\operatorname{deg}(X)} \mathrm{d} \iota_{X}
$$

By construction, $\mathcal{L}_{X}$ super-commutes with the de Rham differential and agrees with the action of vector fields on functions, $\mathcal{L}_{X}(f)=X(f)$. Furthermore, one can also check that the Lie derivative respects the super-Lie algebra of vector fields, generalizing the formulas for even manifolds, such that the following identities hold:

$$
\left[\mathcal{L}_{X}, \mathcal{L}_{Y}\right]=\mathcal{L}_{[X, Y]}, \quad\left[\mathcal{L}_{X}, \iota_{Y}\right]=\iota_{[X, Y]} .
$$

In particular, if $Q$ is a homological vector field, we immediately obtain that

$$
\left[\mathrm{d}, \mathcal{L}_{Q}\right]=\left[\mathcal{L}_{Q}, \mathcal{L}_{Q}\right]=0
$$

As a corollary we conclude that the total space of $T[1] \mathcal{M}$ for a Q-manifold $\mathcal{M}$ is a bigraded manifold supplied with a couple of super-commuting Q-structures which are of degree one w.r.t. the first and the second gradings, respectively. Let us denote the total differential as $Q_{T \mathcal{M}}=\mathrm{d}+\mathcal{L}_{Q}$.

Proposition 3.3 The map $f: \mathcal{M}_{1} \rightarrow T[1] \mathcal{M}_{2}$ is a $Q$-morphism w.r.t. the total $Q$-structure on the target, that is, the following chain property holds:

$$
Q_{1} f^{*}-f^{*} Q_{T \mathcal{M}_{2}}=0 \text {. }
$$

Proof. Taking into account that the 1.h.s. of the equation is always a derivation, it is sufficient to apply it on generators of the algebra of forms (on functions and exact 1-forms). Using Eqs. (3.2) it is then easy to complete the proof.

\footnotetext{
${ }^{6}$ In Eq. (3.6) the brackets indicate graded commutators. For an odd vector field $Q$ the following equations are thus anticommutators, the de Rham differential d anticommutes with the Lie derivative $\mathcal{L}_{Q}$.
} 
3.4 There exists also a conceptually more enlightening proof of the previous proposition. In fact it is easy to convince oneself that except for $\varphi$ all the arrows in the first diagram above are morphisms of Q-manifolds, if we equip the tangent bundles with the respective de Rham differentials. Now the map $f: \mathcal{M}_{1} \rightarrow T[1] \mathcal{M}_{2}$ differs from the (in this sense) Q-morhpism $\varphi_{*} \circ Q_{1}$ by a substraction along the tangent fibers by the respective "value" of $Q_{2}$. This substraction corresponds to $\exp \left(\iota_{Q_{2}}\right)$, a diffeomorphism of $\mathcal{M}_{2}$ generated by the (in $T[1] \mathcal{M}_{2}$ vertical) vector field $\iota_{Q_{2}}$, as one can most easily verify on local coordinate functions. Correspondingly, the new map, which is our $f$, will remain a chain map, if the de Rham differential is twisted/conjugated by $\exp \left(\iota_{Q_{2}}\right)$. We summarize this in the following

Lemma 3.5 The field strength $f: \mathcal{M}_{1} \rightarrow T[1] \mathcal{M}$ of a gauge field $\varphi: \mathcal{M}_{1} \rightarrow \mathcal{M}$ can be defined by the formula $f=\exp \left(\iota_{Q}\right) \circ \varphi_{*} \circ Q_{1}$. It is a Q-morphism w.r.t.

$$
Q_{T \mathcal{M}} \equiv \mathrm{d}+\mathcal{L}_{Q}=\exp \left(\iota_{Q}\right) \mathrm{d} \exp \left(-\iota_{Q}\right)
$$

We remark that $\iota_{Q}$ does not square to zero since $Q$ is odd; still the last equality follows easily from the general formula $\exp (A) B \exp (-A)=\exp \left(a d_{A}\right) B$ (valid for operators $A$ and $B$ that are not both odd) together with (3.6) and $[Q, Q]=0$.

3.6 A natural example of the chain map property of $f$ is provided by the Weil algebra. It is well-known that, if one has a graded morphism from $\Lambda\left(\mathfrak{g}^{*}\right)$ of a Lie algebra $\mathfrak{g}$ to some differential graded commutative algebra $\mathcal{A}$, which is not necessarily a chain map, we can always extend it as a chain map, acting from the Weil algebra $W(g)=S^{\bullet}\left(\mathrm{g}^{*}\right) \otimes \Lambda\left(\mathrm{g}^{*}\right)$ to $\mathcal{A}$. The construction is working as follows: given a graded morphism $\Lambda\left(\mathfrak{g}^{*}\right) \rightarrow \mathcal{A}$, we identify it with some $A$ which belongs to the dg Lie algebra $\mathcal{A} \otimes \mathfrak{g}$, where the differential and the bracket are extended by linearity:

$$
\mathrm{d}(\alpha \otimes X):=\mathrm{d} \alpha \otimes X, \quad[\alpha \otimes X, \beta \otimes Y]:=\alpha \beta \otimes[X, Y]
$$

for any $\alpha, \beta \in \mathcal{A}$ and $X, Y \in \mathfrak{g}$. Defining $F_{A}:=\mathrm{d} A+\frac{1}{2}[A, A]$ (we recognize the curvature of a connection in a trivial bundle as a particular example), the required map $W(g) \rightarrow \mathcal{A}$ is

$$
\Phi \otimes \omega \mapsto \Phi(\underbrace{F_{A}, \ldots, F_{A}}_{q \text { times }}) \omega(\underbrace{A, \ldots, A}_{p \text { times }}), \quad \Phi \in S^{q}\left(\mathrm{~g}^{*}\right), \omega \in \Lambda^{p}\left(\mathrm{~g}^{*}\right) .
$$

One can easily check that the grading and differential in the Weil algebra are chosen in such a way that it becomes isomorphic to $\Omega(\mathfrak{g}[1])$ supplied with the above total differential. Furthermore, the chain map described above is nothing but our map $f$, if $\mathcal{M}_{2}=\mathfrak{g}[1]$ and $\mathcal{A}=C^{\infty}\left(\mathcal{M}_{1}\right) \cdot 7$

3.7 Let $\mathcal{G}$ be a graded Lie subalgebra of vector fields of negative degree on a Q-manifold $(\mathcal{M}, Q)$ which is closed under the derived bracket.

\footnotetext{
${ }^{7}$ In fact, the statement in Proposition 3.3 can be easily adapted to the situation where $C^{\infty}\left(\mathcal{M}_{i}\right)$ are replaced by arbitrary differential graded commutative super algebras and $\varphi$ (or better $\varphi^{*}$ ) by an arbitrary degree preserving morphism.
} 
Definition 3.8 A differential form $\omega \in \Omega(\mathcal{M})$ is called a (generalized) G-basic form, if $\mathcal{L}_{\epsilon}(\omega)=0=\mathcal{L}_{a d_{Q}(\epsilon)}(\omega)$ for each $\epsilon \in \mathcal{G}$. We denote the space of $\mathcal{G}$-basic forms as $\Omega(\mathcal{M})_{\mathcal{G}}$.

$\Omega(\mathcal{M})_{\mathcal{G}}$ is a graded commutative algebra, which is stable with respect to both differentials: The product of two such forms is again a $\mathcal{G}$-basic form. The Lie derivative commutes with the de Rham operator, so the space is invariant with respect to d. Furthermore, by the identities (3.6), one has $\left[\mathcal{L}_{Q}, \mathcal{L}_{\epsilon}\right]=\mathcal{L}_{a d_{Q}(\epsilon)}$ and $\left[\mathcal{L}_{Q}, \mathcal{L}_{a d_{Q}(\epsilon)}\right]=0$, thus $\Omega(\mathcal{M})_{\mathcal{G}}$ is also closed with respect to $\mathcal{L}_{Q}$.

3.9 Now we apply this machinery in a rather straightforward way for the construction of characteristic classes associated to any section (gauge field) of a Q-bundle.

Theorem 3.10 Let $\pi: \mathcal{M} \rightarrow \mathcal{N}$ be a $Q$-bundle with a typical fiber $\mathcal{F}$, a holonomy algebra $\mathcal{G}$, and $\varphi$ a section of $\pi$ (in the graded sense) - cf. Definition 2.8. Then there is a welldefined map in cohomology

$$
H^{p}\left(\Omega(\mathcal{F})_{\mathcal{G}}, Q_{T \mathcal{F}}\right) \rightarrow H^{p}\left(C^{\infty}(\mathcal{N}), Q_{\mathcal{N}}\right),
$$

which does not depend on homotopies of $\varphi$.

Lemma 3.11 Let $\left(\mathcal{M}_{1}, Q_{1}\right)$ and $\left(\mathcal{M}_{2}, Q_{2}\right)$ be $Q$-manifolds, $\varphi$ a morphism $\mathcal{M}_{1} \rightarrow \mathcal{M}_{2}$ of the underlying graded manifolds, and $Y$ a vector field on $\mathcal{M}_{2}$ of degree minus one, generating the inner derivation $X=a d_{Q_{2}}(Y)$. Then the induced variation-the induced infinitesimal gauge transformation-of $f^{*}: \Omega\left(\mathcal{M}_{2}\right) \rightarrow C^{\infty}\left(\mathcal{M}_{1}\right)$ is given by: $\delta_{X} f^{*}=f^{*} \mathcal{L}_{X}$.

Proof. Since $f^{*}$ is a degree preserving map, its variation w.r.t. an infinitesimal flow, generated by a degree zero vector field, is a degree preserving derivation above $f^{*}$. Thus it is sufficient to check the identity on functions $h$ and exact 1-forms $\mathrm{d} h$ over $\mathcal{M}_{2}$. For the first part we can use Proposition 2.9 , since $f^{*}(h)=a^{*}(h)$. Likewisely, using this proposition and eqs. (3.2), we find:

$$
\delta_{X} f^{*} \mathrm{~d} h=\delta_{X} F(h)=Q_{1} \delta_{X} \varphi^{*}(h)-\delta_{X} \varphi^{*} Q_{2}(h)=Q_{1} \varphi^{*} X(h)-\varphi^{*} X Q_{2}(h) .
$$

But, as an inner derivation, $X=a d_{Q_{2}}(Y)$ commutes with $Q_{2}$. Thus, $\delta_{X} f^{*}(\mathrm{~d} h)=F \mathcal{L}_{X}(f)=$ $f^{*} \mathcal{L}_{X}(\mathrm{~d} f)$, which concludes the proof.

Lemma 3.12 Let $\omega \in \Omega\left(\mathcal{M}_{2}\right)_{\mathcal{G}}$, G vector fields on $\mathcal{M}_{2}$ closed w.r.t. the Lie and the derived bracket, and $\omega^{\prime}$ be its trivial extension to the total space of $\pi: \mathcal{M}_{1} \times \mathcal{M}_{2} \rightarrow$ $\mathcal{M}_{1}$. Then $f^{*}\left(\omega^{\prime}\right)$ is invariant under the action of $\exp \left(\mathcal{G}^{\prime}\right)$ on sections, where $\mathcal{G}^{\prime}:=$ $\operatorname{ad}_{Q}\left(C^{\infty}\left(\mathcal{M}_{1}, \mathcal{G}\right)\right) \cap \mathcal{D}^{0}\left(\mathcal{M}_{1} \times \mathcal{M}_{2}\right) .\left(\varphi: \mathcal{M}_{1} \rightarrow \mathcal{M}_{1} \times \mathcal{M}_{2}, Q \equiv Q_{1}+Q_{2}\right)$.

Proof. Let us replace the target manifold $\mathcal{M}_{2}$ in Lemma 3.11 by the total space $\mathcal{M}=$ $\mathcal{M}_{1} \times \mathcal{M}_{2}$ and an arbitrary graded morphism $\varphi$ with a section of $\pi$. Suppose we are given an infinitesimal gauge transformation $X=a d_{Q}(Y)$. Applying Lemma 3.11 to the variation of $f^{*}$ along $X$ and formula (2.9), we obtain:

$$
\delta_{X} f^{*} \omega^{\prime}=f^{*} \mathcal{L}_{a d_{Q}(Y)} \omega^{\prime}=\sum_{j} f^{*}\left(\mathcal{L}_{Q_{1}\left(b^{j}\right) Y_{j}}+(-1)^{\operatorname{deg}\left(b^{j}\right)} \mathcal{L}_{b^{j} a d_{Q_{2}}\left(Y_{j}\right)}\right) \omega^{\prime} .
$$


We are left to prove that $f^{*} \mathcal{L}_{h v} \omega^{\prime}=0$ for each $h \in C^{\infty}\left(\mathcal{M}_{1}\right)$ of degree $\mathrm{q}$ and $v \in \mathcal{D}^{p}\left(\mathcal{M}_{2}\right)$ which obeys $\mathcal{L}_{v} \omega^{\prime}=0$ : By the definition of Lie derivative,

$$
\mathcal{L}_{h v} \omega^{\prime}=\left(\iota_{h v} \mathrm{~d}+(-1)^{p+q} \mathrm{~d} \iota_{h v}\right) \omega^{\prime}=\left((-1)^{p+q} \mathrm{~d} h \wedge \iota_{v}+h \mathcal{L}_{v}\right) \omega^{\prime}=(-1)^{p+q} \mathrm{~d} h \wedge \iota_{v} \omega^{\prime} .
$$

On the other hand, for any section $\varphi$ its field strength $F$ is a vertical derivation:

$$
F(h)=Q_{1} \varphi^{*} \pi^{*}(h)-\varphi^{*}\left(Q_{1}+Q_{2}\right) \pi^{*}(h)=0, \quad \forall h \in C^{\infty}\left(\mathcal{M}_{1}\right),
$$

since $\pi \varphi \equiv \mathrm{Id}$ and $Q \pi^{*}=\pi^{*} Q_{1}$. With this equation and (3.2) we then indeed obtain

$$
f^{*} \mathcal{L}_{h v} \omega^{\prime}=(-1)^{p+q} f^{*}\left(\mathrm{~d} h \wedge \iota_{v} \omega^{\prime}\right)=(-1)^{p+q} F(h) f^{*}\left(\iota_{v} \omega^{\prime}\right) \equiv 0 .
$$

\section{Proof of Theorem 3.10}

Let us fix a trivialization of $\pi$ over an open cover $\mathcal{U}_{i}$. Suppose we are given a section $\varphi$, then there is a family of sections $\varphi_{i}$ over $\mathcal{U}_{i}$ which are the restrictions of $\varphi$. Applying $f_{i}^{*}$ to each trivial extension $\omega_{i}^{\prime}$ of $\omega$ to $\mathcal{U}_{i} \times \mathcal{F}$, we obtain a family of $Q_{1}$-cocycles in $C^{\infty}\left(\mathcal{U}_{i}\right)$, denoted as $\operatorname{char}_{i}(\omega)$. Taking into account that the trivialization is glued by a transition cocycle of gauge transformations belonging to $\exp \left(\mathcal{G}^{\prime}\right)-\mathrm{cf}$. definition 2.8 $\varphi_{i}$ and $\varphi_{j}$ are related by a gauge transformation; thus, applying Lemma 3.12, we obtain $\operatorname{char}_{i}(\omega)=\operatorname{char}_{j}(\omega)$ over $\mathcal{U}_{i} \cap \mathcal{U}_{j}$. Thus one has a global $Q_{1}$-cocycle char $(\omega)$, such that $\operatorname{char}_{i}(\omega)$ is its restriction to $\mathcal{U}_{i}$.

It remains to prove that if $\varphi(t), t \in[0,1]$, is a smooth family of sections, then the cohomology class of the corresponding $\operatorname{char}(\omega)(t)$ does not change. For this purpose we use the same argument as for the usual Chern-Weil formalism. A family of sections $\varphi(t)$ can be thought of as a section $\tilde{\varphi}$ of the following extension of $\pi$ :

$$
\tilde{\pi}: \mathcal{M} \times T[1] I \rightarrow \mathcal{N} \times T[1] I, \quad I=[0,1] .
$$

By construction, for any function $h$ on the total space of $\tilde{\pi}$, written as $h=h_{0}+\mathrm{d} t h_{1}$, where $h_{i} \in C^{\infty}(\mathcal{M} \times I)$, the pull-back with respect to $\tilde{\varphi}$ is $\tilde{\varphi}^{*} h=\varphi^{*}(t) h_{0}+\mathrm{d} t \varphi^{*}(t) h_{1}$. The new field strength operator is $\tilde{F}=F(t)+\mathrm{d} t \partial_{t} \varphi^{*}(t)$. Applying the corresponding characteristic map to $\omega$, we obtain a cocycle on $\mathcal{N} \times T[1] I$, which decomposes as follows:

$$
\widetilde{\operatorname{char}}(\omega)=\operatorname{char}(\omega)(t)+\mathrm{d} t \beta
$$

Using the closedness with respect to $Q_{N}+\mathrm{d}_{I}$, we immediately get the identity

$$
\partial_{t} \operatorname{char}(\omega)(t)=Q_{N} \beta(t),
$$

which implies the invariance of characteristic classes in cohomology:

$$
\operatorname{char}(\omega)(1)-\operatorname{char}(\omega)(0)=Q_{N} \int_{0}^{1} \mathrm{~d} t \beta(t) .
$$


This completes the proof of Theorem 3.10 .

For some purposes like the construction of secondary characteristic classes, it may be useful to display the transgression $\beta(t)$ explicitely. If $q^{\alpha}$ denote (local) graded coordinates on the fiber $\mathcal{F}$, and on the chart $\mathcal{U}_{i}$ we use the notation $\varphi_{i}(t)^{*}\left(q^{\alpha}\right)=: A^{\alpha}(t)$ and $f_{i}(t)^{*}\left(q^{\alpha}\right)=$ : $F^{\alpha}(t)$, denoting the corresponding tower of gauge fields and field strengths, then for $\omega=$ $\frac{1}{p !} \omega_{\alpha_{1} \ldots \alpha_{p}}(x) \mathrm{d} x^{\alpha_{1}} \wedge \ldots \wedge \mathrm{d} x^{\alpha_{p}}$ one finds

$$
\left.\beta(t)\right|_{\mathcal{U}_{i}}=\frac{1}{(p-1) !} \varphi_{i}(t)^{*}\left(\omega_{\alpha_{1} \ldots \alpha_{p}}\right) \partial_{t}\left(A^{\alpha_{1}}(t)\right) \wedge F^{\alpha_{2}}(t) \wedge \ldots \wedge F^{\alpha_{p}}(t)
$$

Note that if $\mathcal{F}$ is a Qk-manifold and $\mathcal{N}=T[1] \Sigma$, then $A^{\alpha} \mathrm{S}$ are in general a tower of differential forms of degree zero up to degree $k$ (and likewise $F^{\alpha}$ s differential forms of degree one up to degree $k+1)$. Also, in general $\omega_{\alpha_{1} \ldots \alpha_{p}}$ will depend on $x^{\alpha}$ and thus its pullback by $\varphi_{i}(t)^{*}$ produce a function in the gauge fields. Clearly, by construction, $\beta(t)$ is well-defined globally on the base $\mathcal{N}$.

3.13 By use of Theorem 3.10 we associate a characteristic class to each gauge field and cohomology class of the subcomplex $\left(\Omega(\mathcal{F})_{\mathcal{G}}, Q_{T \mathcal{F}}\right)$ of $\mathcal{G}$-invariant forms on the fiber.

Proposition 3.14 Suppose c belongs to the kernel in cohomology of the canonical map $\left(\Omega(\mathcal{F})_{\mathcal{G}}, Q_{T \mathcal{F}}\right) \rightarrow\left(\Omega(\mathcal{F}), Q_{T \mathcal{F}}\right)$. Then the corresponding characteristic class is trivial for any trivial $Q$-bundle $\mathcal{M} \rightarrow \mathcal{N}$.

Proof. If the Q-bundle is trivial, then there exists a projection of the total space to the fiber $p_{\mathcal{F}}: \mathcal{M} \rightarrow \mathcal{F}$ which is a Q-morphism. The corresponding characteristic class attached to a gauge field $\varphi$, which is nothing but $f^{*} p_{\mathcal{F}}^{*} c$, is certainly trivial on the base, $f$ being a Q-morphism.

3.15 Bringing the gauge transformation of the field strength $\delta_{a d_{Q_{2}}(Y)} f^{*}$ in Lemma 3.11 into the form $f^{*} \circ a d_{Q_{T \mathcal{M}_{2}}}\left(\mathcal{L}_{Y}\right)$, we observe that it fits the pattern of Proposition 2.9. Given a gauge field $\varphi: \mathcal{M}_{1} \rightarrow \mathcal{M}$ in a Q-bundle $\mathcal{M} \rightarrow \mathcal{M}_{1}$ and an infinitesimal gauge transformation generated by $a d_{Q}(Y), Y$ a vertical vector field of degree minus one, we can canonically associate to these data: A Q-bundle $\left(\widetilde{\mathcal{M}} \rightarrow \mathcal{M}_{1}, \widetilde{Q}\right)$, a gauge field $\widetilde{\varphi}$ which is a true section of the Q-bundle (i.e. indeed in the category of Q-manifolds), and a vertical vector field $\widetilde{Y}$; here $\widetilde{\mathcal{M}}=T[1] \mathcal{M}, \widetilde{Q}=Q_{T \mathcal{M}} \equiv \mathrm{d}+\mathcal{L}_{Q}, \widetilde{\varphi}=f$ (a true section due to Proposition 3.3), and $\widetilde{Y}=\mathcal{L}_{Y}$. Using these identifications we can always, vice versa, recover the primary data. The extended Q-bundle is quite special, however: vector fields in the gauge transformations have a very particular form, generated by $\widetilde{Y}=\left[\iota_{Y}, \mathrm{~d}\right]$ for some $Y$ living on $\mathcal{M}$, and likewisely restricted is the holonomy group $\widetilde{\mathcal{G}} \cong \mathcal{G}$ of $\widetilde{\mathcal{M}}$. Still, in this language, a $\mathcal{G}$-basic form on $\mathcal{F}$ translates into a function on the new fiber $\widetilde{\mathcal{F}}=T[1] \mathcal{F}$ which is annihilated by $\widetilde{\epsilon}$ and $\left[Q_{\widetilde{\mathcal{F}}}, \widetilde{\epsilon}\right]$ where again $\widetilde{\epsilon}$ is of the particular form $\left[\iota_{\epsilon}, \mathrm{d}\right]$ (for some $\epsilon$ living on $\mathcal{F}$ generating the holonomy $\mathcal{G}$ as before). 


\section{Some applications}

4.1 The first evident example is a principal G-bundle over $M$. The corresponding Qbundle is provided by the anchor map $\rho: E[1] \rightarrow T[1] M$ of the associated Atiyah algebroid $E$, cf. examples (3) and (4) in paragraph 1.3 . The holonomy algebra is $\mathfrak{g}$, the Lie algebra of $G$, and the fiber is $\mathfrak{g}[1]$. Using the isomorphism $W(\mathfrak{g}) \simeq \Omega(\mathfrak{g}[1])$ explained in the previous section, we can easily see the isomorphism between the space of basic forms and symmetric $G$-invariant polynomials on $\mathfrak{g}^{*}, \Omega(\mathfrak{g}[1])_{\mathfrak{g}} \simeq S\left(\mathfrak{g}^{*}\right)^{G}$ : An element of $\omega \in \Omega(\mathfrak{g}[1])_{\mathfrak{g}}$ always has the form $\omega=\frac{1}{p !} \omega_{a_{1} \ldots a_{p}} \mathrm{~d} \xi^{a_{1}} \wedge \ldots \wedge \mathrm{d} \xi^{a_{p}}$, if $\xi^{a}$ denote the odd coordinates on $\mathfrak{g}[1]$, with $\omega_{a_{1} \ldots a_{p}}$ being constant, completely symmetric, and adinvariant (following from invariance w.r.t. $\mathcal{L}_{\epsilon}$, graded antisymmetry of $\omega$, and invariance w.r.t. $\mathcal{L}_{a d_{Q}(\epsilon)}$, respectively).

As explained before, a section of $\rho$ is a connection in the principal $G$-bundle, and the construction of characteristic classes in Theorem 3.10 applied to this particular case reproduces the Chern-Weil map. Indeed, with $F^{a}$ denoting the local curvature 2-forms, from the above $\omega$ one obtains

$$
\frac{1}{p !} \omega_{a_{1} \ldots a_{p}} F^{a_{1}} \wedge \ldots \wedge F^{a_{p}}
$$

Likewise, $\beta(t)$ of Eq. (3.15) specified to this case, gives the standard transgression formula in this example.

4.2 Equivariant cohomology and gauging of WZ-terms in sigma models: A near-at-hand extension of the Weil algebra $W(\mathfrak{g})$ is the Weil model of equivariant cohomology. Let $G$ be a Lie group acting on a manifold $M$ and $\rho: \mathfrak{g} \rightarrow \mathcal{D}(M), \mathcal{D}(M) \equiv \Gamma(T M)$, be the corresponding Lie algebra action. The complex one looks at is $C=W(\mathfrak{g}) \otimes \Omega(M)$, equipped with the sum of the previously introduced differential on $W(\mathfrak{g})$ and the de Rham differential on the forms on $M$. In Q-language this is the space of functions on $\widetilde{\mathcal{M}_{2}}:=$ $T[1](\mathfrak{g}[1] \times M)$ and the differential gives rise to a homological vector field on it, which we want to call $Q_{W}$. The g-action is extended in a natural way to this complex. To describe this in the language of section 2 , we need a map from $g$ into vector fields $\mathcal{D}\left(\widehat{\mathcal{M}}_{2}\right) \ni X$ of degree minus one such that $a d_{Q_{W}}(X)$ generates the $g$ action. This is easy to find: Take an element $\epsilon$ in the Lie algebra, it generates canonically a vector field of degree minus one on $\mathfrak{g}[1]$ and thus (by lifting as a Lie derivative) also on $T$ [1] $\mathfrak{g}[1]$. Likewise $\rho(\epsilon)$ gives a vector field on $M$, its contraction $\iota_{\rho(\epsilon)}$ is a degree minus one vector field on $T[1] M$. We denote the sum of these two vector fields by $i_{\epsilon}$. Now $a d_{Q_{W}}\left(i_{\epsilon}\right) \equiv\left[Q_{W}, i_{\epsilon}\right]$ is easily verified to generate the canonical diagonal $\mathfrak{g}$-action on $C^{\infty}\left(\mathcal{M}_{2}\right)$.

An element $\alpha \in C$ is called horizontal if, in the above language, it is annihilated by $i_{\epsilon}$ for all $\epsilon \in \mathfrak{g}$. If, in addition, it is also g-invariant, i.e. also annihilated by $a d_{Q_{W}}\left(i_{\epsilon}\right)$, it is called basic. The space of basic elements in $C$ is denoted by $C_{\mathfrak{g}}$ or by $\Omega(M)_{G}$. Although the space $\widetilde{\mathcal{M}}_{2}$ is of the form $T[1]\left(\mathcal{M}_{2}\right)$ with $\mathcal{M}_{2} \equiv \mathfrak{g}[1] \times M$ and the homological vector field $Q_{W}$ is nothing but the total differential of the Chevalley-Eilenberg differential $Q_{C E} \simeq$ $\mathrm{d}_{\mathfrak{g}}$ on $\mathrm{g}[1]$ extended trivially to $\mathcal{M}_{2}, Q_{W}=\mathrm{d}+\mathcal{L}_{Q_{C E}}$, the notion of basic elements does not agree with the one of Defintion 3.8. This, as we will see also in more detail below, is related to the last remark in the previous section; $\widetilde{\epsilon} \equiv i_{\epsilon}=\mathcal{L}_{\epsilon}+\iota_{\rho(\epsilon)}$ is of the required form 
of a Lie derivative only on $T[1] \mathfrak{g}[1]$, but not also on $T[1] M$.

The reason for this apparent discrepancy is that not all the data have been incorporated properly into the $Q$-structure on $\mathcal{M}_{2}$; in particular, the representation $\rho$ entered only when considering $\widetilde{\epsilon}$. This can be cured easily, however, and will lead us automatically to the so-called Cartan model of equivariant cohomology. The initial data give rise to an action Lie algebroid $E=M \times \mathfrak{g}$ over $M, \rho$ yielding its anchor map. The respective homological vector field of $\mathcal{M}_{2}:=E[1]$ has the form

$$
Q=\rho+Q_{C E}
$$

where we interpreted $\rho$ as an element in $\mathrm{g}^{*} \times \mathcal{D}(M)$, viewing $\mathrm{g}^{*}$ as a linear and thus degree one function on $\mathfrak{g}[1]$. For non-abelian $\mathfrak{g}$, the vector field $\rho$ does not square to zero, but it is easily verified that $\rho^{2}=-\left[Q_{C E}, \rho\right]$, so that indeed $Q^{2}=0.8$ Let us call the canonical lift $\mathrm{d}+\mathcal{L}_{Q}$ of $Q$ to $T[1] \mathcal{M}_{2}$ by $Q_{C}$. Since $Q$ differs from $Q_{C E}$ by the addition of $\rho$ and vertical vector fields on $T[1] \mathcal{M}_{2} \rightarrow \mathcal{M}_{2}$ being contractions of vector fields coming from the base always (super)commute, it follows immediately from (4.1) and Lemma 3.5 that

$$
Q_{W}=\exp \left(-\iota_{\rho}\right) Q_{C} \exp \left(\iota_{\rho}\right)
$$

In this more geometric picture we find that indeed the notion of basic above agrees with Defintion 3.8; $\epsilon$ as before, we see that

$$
\begin{aligned}
\exp \left(-\iota_{\rho}\right) \mathcal{L}_{\epsilon} \exp \left(\iota_{\rho}\right) & =i_{\epsilon} \\
\exp \left(-\iota_{\rho}\right) \mathcal{L}_{a d_{Q}(\epsilon)} \exp \left(\iota_{\rho}\right) & =a d_{Q_{W}}\left(i_{\epsilon}\right)
\end{aligned}
$$

where for the first equality we made use of $\left[\mathcal{L}_{\epsilon}, \iota_{\rho}\right]=\iota_{[\epsilon, \rho]}=\iota_{\rho(\epsilon)}$, which in turn commutes with $\iota_{\rho}$, from which the second one follows immediately on observing $\mathcal{L}_{a d_{Q}(\epsilon)}=\left[Q_{C}, \mathcal{L}_{\epsilon}\right]$. Moreover, elements in $C \cong \Omega(E[1])$ annihilated by $\mathcal{L}_{e}$ are easily seen to be elements in $S^{\bullet}\left(\mathfrak{g}^{*}\right) \otimes \Omega(M)$. On the other hand, the map $\rho$ is equivariant and thus the respective homological vector field $\rho$ is g-invariant. This implies that $a d_{Q_{W}}\left(i_{\epsilon}\right)$ commutes with $\exp \left(\iota_{\rho}\right)$, which in addition to eq. (4.4) yields the equality $\mathcal{L}_{a d_{Q}(\epsilon)}=a d_{Q_{W}}\left(i_{\epsilon}\right)$. Thus annihilation by $\mathcal{L}_{a d_{Q}(\epsilon)}$ implies $\mathfrak{g}$-invariance and basic differential forms on the action Lie algebroid $E[1]$ following Definition 3.8 correspond precisely to elements in $\left(S^{\bullet}\left(\mathfrak{g}^{*}\right) \otimes \Omega(M)\right)^{G}$, which is the Cartan model of equivariant cohomology.

This is now also the right language and departure point for the discussion of gauging of WZ-terms in sigma models (cf. also [4]). Let d-dimensional "spacetime" be the boundary of $N$ and consider the space of maps $X: N \rightarrow M$ as (part of) the "fields" of the sigma model. A WZ-term then is induced by a closed (d+1)-form $H$ on $M, S_{W Z}[X]=$ $\int_{N} X^{*} H$. G-invariance of $H$ yields $S_{W Z}$ invariant under "rigid" G-transformations, but not under "local" ones, i.e. where the transformation parameters are permitted to vary along $N$. To capture this fact in the present framework, we extend $X$ trivially to a map

\footnotetext{
${ }^{8}$ In local coordinates $x^{i}, \xi^{a}$ on $\mathcal{M}_{2}=M \times \mathfrak{g}[1]$, one recognizes in $Q=\xi^{a} \rho_{a}-\frac{1}{2} \xi^{b} \xi^{c} C_{b c}^{a} \partial_{a}$, where $C_{b c}^{a}$ are the structure constants of $\mathfrak{g}$ in the basis $\xi_{a}$ dual to $\xi^{a}$ and $\rho_{a} \equiv \rho\left(\xi_{a}\right)$, one recognizes the standard YM-type BRST charge. Although not inspired by [8], the considerations in this paragraph partially parallel, and possibly also simplify and highlight, those of that paper.
} 
$\varphi_{0}:\left(T[1] N, Q_{d R}\right) \rightarrow(M, 0)$; the WZ-term then can also be written as $S_{W Z}\left[\varphi_{0}\right]=\int_{N} f_{0}^{*} H$, where $f_{0}=X_{*}: T[1] N \rightarrow T[1] M$ is the "field strength" of $\varphi_{0}$. In this simple case the chain map property of Proposition 3.3 reduces to the wellknown fact that the pullback map $X^{*}$ commutes with the de Rham differential.

The above mentioned "local G-transformations" will now become gauge transformations in the trivial Q-bundle $T[1] N \times M \rightarrow T[1] N$ as discussed in section 2, Indeed, the representation $\rho$ singles out a Lie subalgebra $\mathfrak{g} \subset \mathcal{D}(M)$ of symmetries on $M$ (there are certainy no inner infinitesimal symmetries on $(M, 0))$; thus functions on $T[1] N$ with values in this Lie subalgebra of degree zero vector fields on $M$ can be used as a proper replacement of the infinitesimal gauge Lie algebra $\cong \mathcal{G}^{\prime} \ni X$ in this context. Its lift to the field strength, $\delta_{X} f_{0}^{*}=f_{0}^{*} \mathcal{L}_{X}$ (cf. Lemma 3.11), produces the correct transformation. Note that now $\varphi_{0}$ is considered as a section in the above trivial Q-bundle (and $H$ is extended in a likewise manner to the trivial bundle); only like this [2] we can accomodate for the $N$-dependence of the infinitesimal generator $X$ of the transformations, $X=\epsilon^{a} \rho_{a}$, in the notation of the previous footnote, with $\epsilon^{a}$ an arbitrary function on $N$. Clearly, $S_{W z}\left[\varphi_{0}\right]$ is not invariant under any such gauge transformation, since the Lie derivative contains the de Rham differential on $N$ and one obtains $f_{0}^{*} \mathcal{L}_{X} H=\mathrm{d} \epsilon^{a} \wedge X^{*}\left(\iota_{\rho_{a}} H\right)+\epsilon^{a} X^{*}\left(\mathcal{L}_{\rho_{a}} H\right)$, where only the second term vanishes by invariance of $H .9$

To cure this one wants to introduce extra gauge field dependent terms into the action functional, i.e. terms depending on additional g-valued 1-forms $A^{a}$ on (the boundary of) $N$. This is possible if $H$ permits a G-equivariantly closed extension $\hat{H}$, cf. also [4]. In our picture the resulting invariant action functional is now easy to obtain: We simply replace $(M, 0)$ in all of the constructions above by the action Lie algebroid $E[1]=M \times \mathfrak{g}[1]$ with its canonical differential, eq. (4.1). $\varphi$ now is a section in the trivial Q-bundle $T[1] N \times$ $E[1] \rightarrow T[1] N$ and $\hat{H}$ extended trivially from $\Omega(E[1])$ to a differential form on the total Q-bundle (analogous to the case of $H$ before). Gauge transformations are now inner right away, they are generated by $\epsilon \mathrm{S}$ as described in the above Cartan-type model of equivariant cohomology, tensored with functions on the base. Gauge invariance of $f^{*} \hat{H}$ now follows at once from the general result of Lemma 3.12. From Proposition 3.3, moreover, it follows immediately that $f^{*} \hat{H}$ is closed and that $f^{*} \hat{H}-f^{*} H$ is exact; thus, the additional gauge fields, corresponding to a degree preserving map from $T[1] N$ to $\mathfrak{g}[1]$, indeed need to be defined over the boundary of $N$ only.

The formalism developed in this paper is certainly aimed at also more general type of gauge theories as those stemming from a structural Lie group like in this paragraph. We intend to make this kind of application more explicit elsewhere, focusing in the present draft mainly on the issue of (generalized) characteristic classes 10

4.3 Another application of the considerations of the previous section is the following

\footnotetext{
${ }^{9}$ If one considers $f_{0}$ as a "gauge field" $\widetilde{\varphi_{0}}$ itself, cf. the discussion at the end of the previous section, the gauge transformations become inner and this becomes a special case of eq. (2.9). However, the field $\widetilde{\varphi_{0}}$ is restricte to derive from the "field strength" of some $\varphi_{0}$ and it is also this perspective that now permits to discuss the gauging of the WZ term in a concise manner.

${ }^{10}$ But cf. also [2, 21, 5], as well as the following paragraph, used to prepare grounds for characteristic classes associated to "PQ"-bundles in the subsequent paragraph 4.7 below.
} 
Theorem 4.4 Let $(\mathcal{S}, \omega)$ be a symplectic $Q p$-manifold, $p \in \mathbb{N}+$, as in example (3) of paragraph 1.2, $N$ a $(p+2)$-dimensional manifold with boundary $\partial N=\Sigma$, and $\varphi$ a (degree preserving) map from $T[1] N$ to $\mathcal{S}$. Then

$$
\int_{N} f^{*} \omega=S_{\Sigma,(c l)}^{A K S Z}
$$

where $S_{\Sigma,(c l)}^{A K S Z}$ is the (classical part of the) topological sigma model on the ( $\left.p+1\right)$-dimensional $\Sigma$ obtained by the AKSZ-method [1].

If $\varphi$ is a gauge field in the sense of this paper, i.e., being a degree preserving map, $\varphi$ has degree zero, one obtains the classical part of the topological action. Permitting all possible degrees of $\varphi$, one gets its BV extension, satisfying the classical BV-master equation, i.e. squaring to zero w.r.t. the BV bracket. For $p=1$ the action reproduces the Poisson sigma model [19, 7], for $p=2$ one obtains the Courant sigma model [6, 16, 17]; the formula above holds for arbitrary dimensions.

Before proving this, we make some general remarks on PQ-manifolds with $p>011$. This is a $\mathrm{Q}$-manifold $\mathcal{S}$ equipped with a compatible symplectic form $\omega$ of degree p, i.e. it obeys $\mathcal{L}_{\xi} \omega=p \omega$, where $\xi$ is the Euler field which provides the $\mathbb{Z}$-grading on $\mathcal{S}$. Nondegeneracy of $\omega$ implies that $\mathcal{S}$ has degree at most $p$ (if $\mathcal{S}$ has a nontrivial body, i.e. its algebra of functions has degree zero elements, this bound is also necessarily saturated); since a lower-degree Q-manifold can also be considered as a degenerate Qp-manifold for some higher $\mathrm{p}$, we will follow the convention that PQ-manifolds of degree $\mathrm{p}$ imply that $\omega$ has degree p. E.g. given a quadratic Lie algebra $(\mathfrak{g}, \kappa), \kappa$ denoting the adinvariant scalar product, we will view $(\mathfrak{g}[1], \omega), \omega \sim \kappa$, as a degree 2 PQ-manifold. For $p>0$ the symplectic form is necessarily exact: By (3.5), one has $\mathrm{d} \iota_{\xi} \omega=p \omega$, thus $\omega=\mathrm{d} \alpha$, where $\alpha=\frac{1}{p} \iota_{\xi} \omega$. For any function $h$ of degree $q$ on $\mathcal{S}$ we define its Hamiltonian vector field $X_{h}$ of degree $q-p$ by the formula: $\iota_{X_{h}} \omega=(-1)^{q+1} \mathrm{~d} h$. Then Hamiltonian vector fields satisfy the known relations from the ungraded case:

$$
\left[X_{h_{1}}, X_{h_{2}}\right]=X_{\left\{h_{1}, h_{2}\right\}}
$$

where $\{\cdot, \cdot\}$ is the induced Poisson bracket of degree $-p$. Now, using the relations (3.6), we can easily verify that compatibility of $\omega$ with $Q, \mathcal{L}_{Q} \omega=0$, implies that $Q$ is always Hamiltonian: $i_{Q} \omega=(-1)^{p} \mathrm{~d} Q$ with the Hamiltonian function $Q$ of degree $\mathrm{p}+1$. We summarize this in the following

Lemma 4.5 For a Qp-manifold with compatible sympectic form w one has

$$
\omega=\mathrm{d} \alpha, \alpha \equiv \frac{1}{p} \iota_{\xi} \omega, \quad Q=X_{Q}, Q \equiv \frac{p}{p+1}(-1)^{p} \iota_{Q} \alpha
$$

where $\xi$ is the Euler vector field on $\mathcal{S}$ and the Hamiltonian $Q$ of the homological vector field $Q$ satisfies the master equation $\{Q, Q\}=0$.

\footnotetext{
${ }^{11}$ This assumption will be kept without further mention.
} 
The last statement follows from eq. (4.6) and the fact that in positive degrees the only constant is zero ( $Q$ has degree $p+1$ and thus $\{Q, Q\}$ degree $p+2)$. From the above we derive

Lemma 4.6 The following transgression formula holds:

$$
\omega=Q_{T \mathcal{S}}(\hat{\alpha}), \quad \hat{\alpha}=\alpha+\frac{(-1)^{p}}{p} Q \equiv \frac{1}{p}\left(1+\frac{1}{p+1} \iota_{Q}\right) \iota_{\xi} \omega .
$$

Proof. With Lemma 4.5 and eq. (3.9) we have $\exp \left(\iota_{Q}\right) \omega=Q_{T S} \exp \left(\iota_{Q}\right) \alpha$. The 1.h.s. of this equation is $\omega+(-1)^{p} \mathrm{~d} Q$, by defintion of the Hamiltonian for $Q$ and the fact that $\iota_{Q} \iota_{Q} \omega$ vanishes on behalf of $\{Q, Q\}=0$. The master equation also implies that $\mathrm{d} Q=Q_{T S} Q$, from which we now can derive easily the wished for transgression formula.

\section{Proof of Theorem 4.4}

With Lemma 4.6 one obtains $f^{*} \omega=\mathrm{d} f^{*} \hat{\alpha}$, where the chain property Prop. 3.3 of $f$ has been used and the fact that $Q_{1}$ is just the de Rham differential on $N$ here. Using Stokes theorem, we are thus left with showing that $f^{*} \hat{\alpha}$ indeed agrees with the AKSZ action (where we can replace $N$ by its boundary $\Sigma$ now). By means of the formulas (3.2), one can convince oneself that for any 1-form $\alpha$ on $\mathcal{S}$ one has

$$
f^{*} \alpha=\left(\iota_{Q_{T[1] \Sigma}} \varphi^{*}-\varphi^{*} \iota_{Q_{S}}\right) \alpha .
$$

Together with the first equation in (3.2) and the second equation in (4.7), this implies

$$
f^{*} \hat{\alpha}=\iota_{\mathrm{d}_{\Sigma}} \varphi^{*} \alpha+(-1)^{p+1} \varphi^{*}(Q),
$$

where $Q_{T[1] \Sigma}=\mathrm{d}_{\Sigma}$ has been used. This expression agrees precisely with the one found in [17] for the AKSZ sigma model, which thus completes the proof.

4.7 Q-bundles with PQ-manifolds $(\mathcal{S}, \omega)$ as fibers are natural candidates for a non-trivial characterisitic class along the lines of Theorem 3.10. As we saw above, the symplectic form $\omega$ itself is closed w.r.t. the total differential $Q_{T S}$ on the fiber and for the holonomy group $\mathcal{G}$ the Lie algebra of (all or a closed subset of the) Hamiltonian vector fields of negative degree lends itself naturally, since $\omega$ is then also basic w.r.t. $G$ 12 A PQbundle (a Q-bundle with PQ-fibers) thus carries a canonical characteristic class. For the Atiyah algebroid of a principal $G$-bundle (cf. example (3) and (4) in paragraph 1.3) where the Lie algebra $g$ of $G$ is equipped with a non-degenerate invariant symmetric form, the corresponding PQ-bundle has a typical fiber $\mathfrak{g}[1]$ together with a degree $p=2$ symplectic form $\omega$ provided by the invariant metric. The canonical characteristic class is nothing but the second Chern class (or the first Pontrjagin class) of the

\footnotetext{
${ }^{12}$ In fact, $\omega$ is even exact within the complex $\left(\Omega(\mathcal{S}), Q_{T S}\right)$, cf. Lemma 4.6 above; however, in general it will fail to be exact within the restricted complex $\left(\Omega(\mathcal{S})_{\mathcal{G}}, Q_{T S}\right)$ of basic forms. This happens already for the standard characteristic classes, paragraph 4.1 above, where the unrestricted cohomology, being isomorphic to deRham cohomology on the Lie algebra, cf. Lemma 3.5 is obviously trivial. It is also in this context where Proposition 3.14 comes into play.
} 
principal $G$-bundle and the Theorem 4.4 simply gives the well-known local statement: "Second Chern form $=\mathrm{d}($ Chern-Simons form $)$ ". For an arbitrary PQ-bundle over a base $T[1] N$ for a smooth manifold $N$ one thus has a straightforward generalization of the second Chern class, which is a $p+2$ cohomology class for a degree $p$ QP-fiber.

For example, in the case of $p=1$ a typical fiber $\mathcal{F}$ is necessarily of the form $T^{*}[1] P$ for some Poisson manifold $(P,\{\cdot, \cdot\})$. Like any Q1-manifold, the total space corresponds to a Lie algebroid $E$ living over some base manifold $M$. Thus the Q-bundle has the form

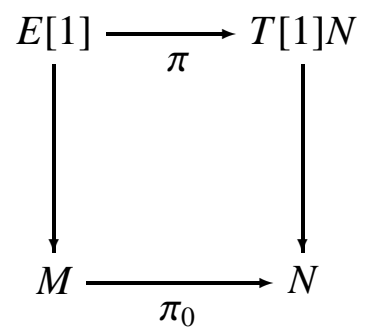

covering an ordinary bundle $\pi_{0}: M \rightarrow N$ whose typical fiber is $P$. It can be shown that this bundle $\pi_{0}$ is a Hamiltonian Poisson fibration, i.e. the Lie algebra of its holonomy group consist of Hamiltonian vector fields of the Poisson manifold $P$. As we will show in detail in a separate note [10], the 3 -form class on $N$ that one obtains in this way does not depend on the chosen gauge field $\varphi: T[1] N \rightarrow E[1]$. Moreover, it constitutes an obstruction to lifting the bundle $\pi_{0}$ to one where the transition cocycle takes values in a group whose Lie algebra is $\left(C^{\infty}(P),\{\cdot, \cdot\}\right)$ - while the existence of a lift to the case of Hamiltonian functions modulo constants is already guaranteed by the existence of the Q-bundle $\pi$ covering $\pi_{0}$.

4.8 Suppose we are given a bundle $p: \mathcal{M} \rightarrow \mathcal{N}$ in the category of Q-manifolds which, in general, is not locally trivial. Let us denote by $\Omega_{+}(\mathcal{N})$ the ideal of all differential forms the order of which as a differential form is greater or equal to one, and by $\mathcal{I}$ the ideal in $\Omega(\mathcal{M})$ generated by the pullback $p^{*} \Omega_{+}(\mathcal{N})$. It is clear that $\mathcal{I}$ is closed with respect to both differentials $d$ and $\mathcal{L}_{Q}$, and thus with respect to the total differential $Q_{T \mathcal{M}}$. For each gauge field $\varphi: \mathcal{N} \rightarrow \mathcal{M}$ the corresponding map $f^{*}: \Omega(\mathcal{M}) \rightarrow C^{\infty}(\mathcal{N})$ vanishes on $\mathcal{I}$ (this fact was used in the proof of Lemma 3.12). Therefore we have a well-defined chain map of complexes

$$
\left(\Omega(\mathcal{M}) / \mathcal{I}, Q_{T \mathcal{M}}\right) \stackrel{f^{*}}{\rightarrow}\left(C^{\infty}(\mathcal{N}), Q_{\mathcal{N}}\right)
$$

which induces a map in cohomology. The conditions of local triviality and gluing by use of $\exp \left(\mathcal{G}^{\prime}\right)$-valued transition cocycle used in the previous section gives a natural chain map of complexes

$$
\left(\Omega(\mathcal{F})_{\mathcal{G}}, Q_{T \mathcal{F}}\right) \rightarrow\left(\Omega(\mathcal{M}) / \mathcal{I}, Q_{T \mathcal{M}}\right),
$$

where $\mathcal{F}$ is the typical fiber of $p$ (Lemma 3.12), the composition of which with (4.11) determines the characteristic map (3.11) in Theorem 3.10.

Let us consider an exact sequence of Lie algebras

$$
0 \rightarrow \mathfrak{h} \rightarrow \mathfrak{g} \stackrel{p}{\rightarrow} \mathfrak{g}_{0} \rightarrow 0,
$$


which defines a locally non-trivial Q-bundle $p: \mathfrak{g}[1] \rightarrow \mathfrak{g}_{0}[1]$ (we denote the induced projection by the same letter $p$ ). The non-triviality means precisely that the exact sequence (4.13) does not split in the category of Lie algebras; here local triviality would imply the global one. The chain map (4.11) provided by a gauge field $\varphi: \mathfrak{g}_{0} \rightarrow \mathfrak{g}$ in these settings induces a chain map $S^{\bullet}\left(\mathfrak{h}^{*}\right) \otimes \Lambda^{\bullet}\left(\mathfrak{g}^{*}\right) \rightarrow \Lambda^{\bullet}\left(\mathfrak{g}_{0}^{*}\right)$. Note that here we used a natural identification induced by the embedding $\iota: \mathfrak{h} \rightarrow \mathfrak{g}$. This chain map, composed with the embedding $S^{\bullet}\left(\mathfrak{h}^{*}\right)^{G} \rightarrow S^{\bullet}\left(\mathfrak{h}^{*}\right) \otimes \Lambda^{\bullet}\left(\mathfrak{g}^{*}\right)$, gives nothing but the characteristic map of exact triples of Lie algebras of Lecomte [11]. For the definition of $S^{\bullet}\left(\mathfrak{h}^{*}\right)^{G}$ one again makes use of the embedding $\iota$. It is this embedding that induces the proper replacement of (4.12).

The complete construction of the characteristic map of Lecomte involves twistings by representations $(V, \rho)$ of $\mathfrak{g}_{0}$, such that the result is taking values in $H^{\bullet}\left(\mathfrak{g}_{0}, V\right)$. In the picture above we need to replace $\mathfrak{g}_{0}$ and $\mathfrak{g}$ with the semidirect products $\tilde{\mathfrak{g}}_{0}=\mathfrak{g}_{0} \ltimes_{\rho^{*}} V^{*}$ and $\tilde{\mathfrak{g}}=\mathfrak{g} \ltimes_{\rho^{*} \circ p} V^{*}$, respectively, which allows extending the sequence (4.13) canonically to:

$$
0 \rightarrow \mathfrak{h} \rightarrow \tilde{\mathfrak{g}} \stackrel{\tilde{p}}{\rightarrow} \tilde{\mathfrak{g}}_{0} \rightarrow 0
$$

Applying the characteristic map $S^{\bullet}\left(\mathfrak{h}^{*}\right)^{G} \rightarrow H^{\bullet}\left(\tilde{\mathfrak{g}}_{0}\right)$ and using the natural isomorphism $H^{q}\left(\tilde{\mathfrak{g}}_{0}\right)=\oplus_{k+l=q} H^{k}\left(\mathfrak{g}_{0}, \Lambda^{l} V\right)$, we immediately obtain the characteristic classes of Lecomte taking values in all exterior powers of the representation $\rho$.

It may be interesting to find more general conditions for a Q-bundle (weaker than those in Definition 2.8) providing an extended version of the map (4.12) which includes simultaneously the construction of Theorem 3.11 and the Lecomte characteristic classes.

\section{Acknowledgments}

The work of A.K. was partially supported by the research grant R1F105L10 of the University of Luxembourg. The authors greatly acknowledge the possibility to work on the completion of the article at the Erwin Schrödinger Institute during the programm "Poisson sigma models, Lie algebroids, deformations, and higher analogues". T.S. is grateful to T. Masson for drawing our attention to Ref. [11].

\section{References}

[1] M. Alexandrov, M. Kontsevich, A. Schwartz, and O. Zaboronsky. The geometry of the master equation and topological quantum field theory. Int. J. Mod. Phys., A12: 1405-1430, 1997.

[2] M. Bojowald, A. Kotov, T. Strobl. Lie algebroid morphisms, Poisson Sigma Models, and off-shell closed gauge symmetries. J.Geom.Phys., 54: 400-426, 2004.

[3] H. Cartan. Notions d'algèbre différentielle; application aux groupes de Lie et aux variétés où opère un groupe de Lie. Colloque de topologie (espaces fibrés) (Bruxelles), Georges Thone, Liège, Masson et Cie., Paris., 1950. 
[4] J. Figueroa-O'Farrill, S. Stanciu. Gauged Wess-Zumino terms and equivariant cohomology. Physics Letters B, 341(2), 153-159, 1994.

[5] M. Gruetzmann, T. Strobl, General Yang-Mills type gauge theories for p-form gauge fields: A physics approach. In preparation.

[6] N. Ikeda. Chern-Simons gauge theory coupled with BF theory. Int. J. Mod. Phys., A18:2689-2702, 2003. hep-th/0203043.

[7] N. Ikeda. Two-dimensional gravity and nonlinear gauge theory. Ann. Phys., 235:435-464, 1994.

[8] J. Kalkman. BRST model for equivariant cohomology and representatives for the equivariant Thom class. Commun. Math. Phys., 153: 447-463, 1993.

[9] Y. Kosmann-Schwarzbach. Derived brackets. Lett.Math.Phys., 69: 61-87, 2004.

[10] A. Kotov, T. Strobl. In preparation, 2007.

[11] P. Lecomte. Sur la suite exacte canonique associée à un fibré principal. Bull. Soc. Math. France, 113: 259-271, 1985.

[12] D. Leites. Introduction to the theory of supermanifolds. Russian Math. Surveys, 35 (1): 3-57, 1980.

[13] K. C. H. Mackenzie. Double Lie algebroids and the double of a Lie bialgebroid. arXiv:math.DG/9808081

[14] K. C. H. Mackenzie. Lie algebroids and Lie pseudoalgebras. Bull. London Math. Soc., 27 (2), 97-147, 1995.

[15] R. A. Mehta. Q-algebroids and their cohomology. arXiv:math.DG/0703234.

[16] Jae-Suk Park. Topological open p-branes. Symplectic geometry and mirror symmetry (Seoul, 2000), 311-384, World Sci. Publishing, River Edge, NJ, 2001. hep-th/0012141.

[17] D. Roytenberg. AKSZ-BV Formalism and Courant Algebroid-induced Topological Field Theories. arXiv:hep-th/0608150.

[18] D. Roytenberg. On the structure of graded symplectic supermanifolds and Courant algebroids. Quantization, Poisson Brackets and Beyond, Theodore Voronov (ed.), Contemp. Math., Vol. 315, Amer. Math. Soc., Providence, RI, 2002. arXiv:math.SG/0203110.

[19] P. Schaller, T. Strobl. Poisson structure induced (topological) field theories. Mod. Phys. Lett., A9:3129-3136, 1994.

[20] A. Schwartz. Semiclassical approximation in Batalin-Vilkovisky formalism. Commun. Math. Phys., 158 (2): 373-396, 1993. 
[21] T. Strobl. Algebroid Yang-Mills theories. Phys. Rev. Lett., 93, 211601, 2004.

[22] A. Vaintrob. Lie algebroids and homological vector fields. Uspekhi Matem. Nauk., 52(2): 428-429, 1997.

[23] T. Voronov. Higher derived brackets and homotopy algebras. J. Pure and Appl. Algebra., 202 (1-3): 133-153, 2005.

[24] T. Voronov. Mackenzie theory and Q-manifolds. arXiv:math.DG/0608111. 